\title{
Water-stabilized plasma generators
}

\author{
Milan Hrabovský \\ Institute of Plasma Physics, Academy of Sciences of CR, 18221 Prague 8, \\ Czech Republic
}

\begin{abstract}
Plasma torches with liquid-stabilized arcs provide an alternative to commonly used sources of thermal plasmas based on gas-stabilized arcs or RF discharges. Relatively long arc columns can be stabilized by liquid walls under very low mass flow rates through the torch chamber. This leads to high values of plasma temperature and enthalpy. In water-stabilized torches, oxygen-hydrogen plasma jet is produced with extremely high plasma enthalpy and flow velocity. Plasma torches with water-stabilized arc provide special performance characteristics in some plasma processing applications like plasma spraying or waste treatment. Physical processes which determine properties of generated plasma are discussed and basic characteristics of water plasma torch are presented in the paper.
\end{abstract}

\section{INTRODUCTION}

Thermal plasmas are commonly generated in inductively coupled discharges or in electric arcs which are stabilized by flowing gas. Typically, inductively coupled plasma torches are characterized by plasma temperatures from $6000 \mathrm{~K}$ to $10000 \mathrm{~K}$. Averaged temperatures in torches with non-transferred electric arcs with gas stabilization are somewhat higher, usually in the range from $8000 \mathrm{~K}$ to $14000 \mathrm{~K}$. Average plasma enthalpies, determined as ratio of the useful power of plasma generator to the flow rate of plasma forming gas, vary from 1 to $100 \mathrm{MJ} / \mathrm{kg}$. Further increase of plasma temperatures and enthalpies is limited by the fact that flowing gas protects the arc chamber walls from thermal overloading and thus a minimum possible gas flow rate exists for given arc power. Higher thermal loading is possible if the walls are created by liquid and an arc is stabilized by wall evaporation. Thus, liquid-stabilized arcs can be utilized as sources of thermal plasmas with high temperatures and enthalpies.

The electric arc with the stabilization of arc column by water vortex was first described more than seventy years ago by Gerdien and Lotz (ref. 1,2). Basic experimental investigations of the water stabilized arcs were performed in the fifties. Maecker et al. (ref. 3,4) measured basic electric characteristics of the arc and studied effect of the length and diameter of the stabilizing channel. Several investigators measured arc plasma temperatures using methods of emission spectroscopy (ref. 5,6,7). All authors reported very high plasma temperatures in the arc column with maximum about $50000 \mathrm{~K}$ in the centerline position. The principle of arc stabilization by water vortex was utilized in the plasma torch designed for plasma spraying and cutting (ref. 8). Despite of the potential of achieving extreme performance characteristics in plasma processing applications the development of liquid-stabilized torches has been limited, especially due to their more complex structure and due to lack of understanding of physical processes in the arc which is necessary for improvement of the torch design.

This paper presents results of investigation of water-stabilized plasma torches which has been established several years ago with the aim of better understanding of arc processes, determination of characteristics of generated plasma jet and further development of plasma torch design. Differences of water and gas stabilized generators are discussed and experimental characteristics of arc and of the generated plasma jet are presented. 


\section{ARC COLUMN STABILIZED BY WATER VORTEX}

\section{Principle of stabilization of arc by liquid}

The schematic picture of a water-stabilized arc is shown in Fig. 1. An arc is ignited in the center of water vortex which is created in an arc chamber with tangential injection of water. Water flows over the segment

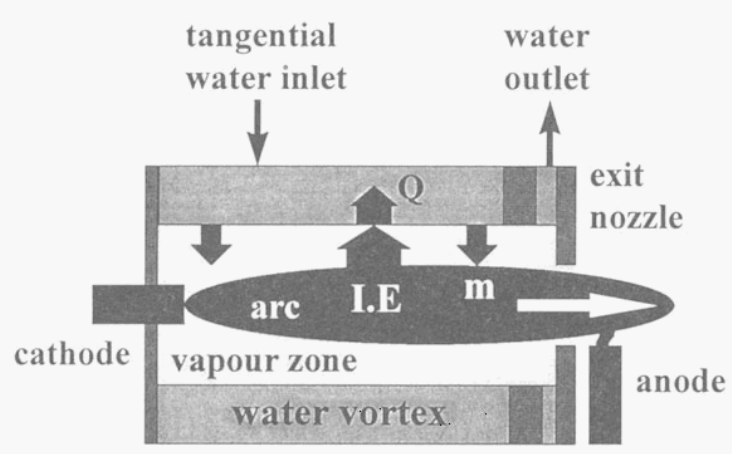

Fig. 1 Schematics of water-stabilized arc which determines inner diameter of the vortex into the exhaust slot. Energy dissipated in the conducting arc core by Joule heating is transported to the inner surface of water vortex by radiation, heat conduction and turbulent transfer. Evaporation of water and heating and ionization of vapour are principal mechanisms that produce arc plasma. Evaporation rate $\mathrm{m}$ is determined by a fraction of total power reaching the water surface. The other part of transferred energy is absorbed in a vapour sheath between the arc column and water surface where it causes heating and ionization of vapour. Power loss $Q$ is given by a part of energy which is transferred into the water volume.

\section{Effect of properties of plasma gas and principle of arc stabilization on arc characteristics}

Effect of material properties of plasma medium and torch chamber dimensions on arc characteristics can be analyzed on the basis of integral energy balance equation of cylindrical arc column (ref. 9)

$$
\frac{\partial\left(\overline{\rho v_{z} h} A\right)}{\partial z}-m \cdot h(R)=A \bar{\sigma} E^{2}+2 \pi R\left(k \frac{\partial T}{\partial r}\right)_{r=R}-4 \pi \overline{\varepsilon_{n}} A
$$

where $\rho$ is plasma density, $v_{z}$ axial velocity, $h$ enthalpy, $\sigma$ electric conductivity, $k$ thermal conductivity, $T$ temperature, $\varepsilon_{n}$ net emission coefficient representing power loss due to radiation and $E$ electric field intensity. $\mathrm{A}$ is cross section of the arc chamber and $\mathrm{R}$ its radius. Quantities averaged over the cross section $A$ are defined by the equation $\bar{X}=\frac{1}{\pi R^{2}} \int_{0}^{R} 2 \pi r X d r$. Simple equation can be obtained if derivatives in equation (1) are approximated as $\frac{\partial\left(\overline{\rho v_{z} h} A\right)}{\partial z}=\frac{\overline{\rho v_{z} h} A}{L}$ and $\left(k \frac{\partial T}{\partial t}\right)_{r=R}=\left(\frac{\partial S}{\partial r}\right)_{r=R}=-\frac{\bar{S}}{R}$, where heat flux potential $S$ is defined as $S=\int_{T_{0}}^{T} k d T$ and $L$ is arc length. Enthalpy $h(R)$ of vapor flowing from the wall into the arc chamber corresponds to the boiling temperature of water $T_{B}$ and can be put equal to zcro $h(R)=h\left(T_{B}\right)=0$. Following two equations for electric field intensity and arc current can be then derived

$$
\begin{aligned}
& E=\frac{1}{R \sqrt{\pi \bar{\sigma}}} \sqrt{\frac{G \bar{h}}{L}+2 \pi \bar{S}+4 \pi^{2} R^{2} \overline{\varepsilon_{n}}} \\
& I=R \sqrt{\pi \bar{\sigma}} \sqrt{\frac{G \bar{h}}{L}+2 \pi \bar{S}+4 \pi^{2} R^{2} \overline{\varepsilon_{n}}}
\end{aligned}
$$

where arc current $I=E \int_{0}^{R} 2 \pi r \sigma d r=\pi R^{2} \bar{\sigma} E$ and total mass flow rate $G=\int_{0}^{R} 2 \pi r \rho v_{z} d r=\pi R^{2} \overline{\rho v_{z}}$. 
The same equations can be written for arcs in plasma torches with gas stabilization, where $m=0$. On the right hand sides of the equations (2) and (3) are physical quantities dependent on temperature, geometrical design parameters $L, R$ and total mass flow rate $G$. Thus, relations between electric characteristics of the $\operatorname{arc} I, E$ and averaged plasma temperature can be evaluated from the equations for given values of the ratio $\mathrm{G} / \mathrm{L}$ and for given arc chamber radius $R$.

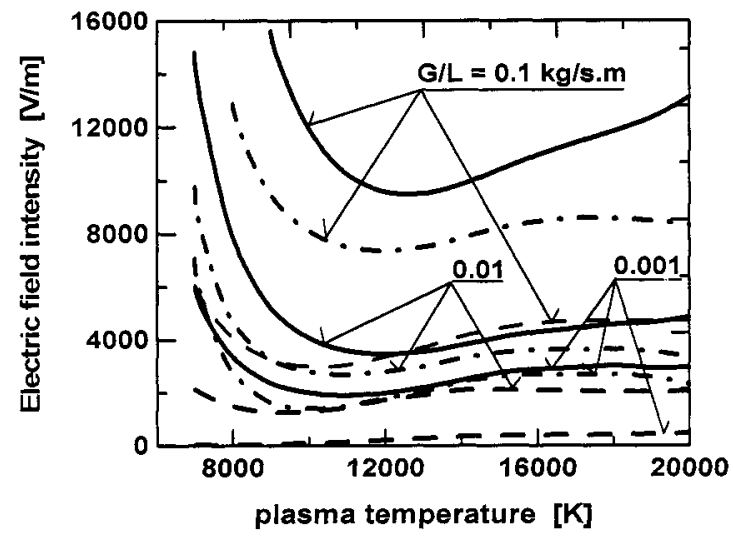

Fig. 2 Electric field intensity in the arc column with radius $\mathrm{R}=3 \mathrm{~mm}$ for different ratios of gas flow rate $\mathrm{G}$ to the arc length $\mathrm{L}$ for arc in water (full line), nitrogen (dash-and-dot line) and argon (dash line).

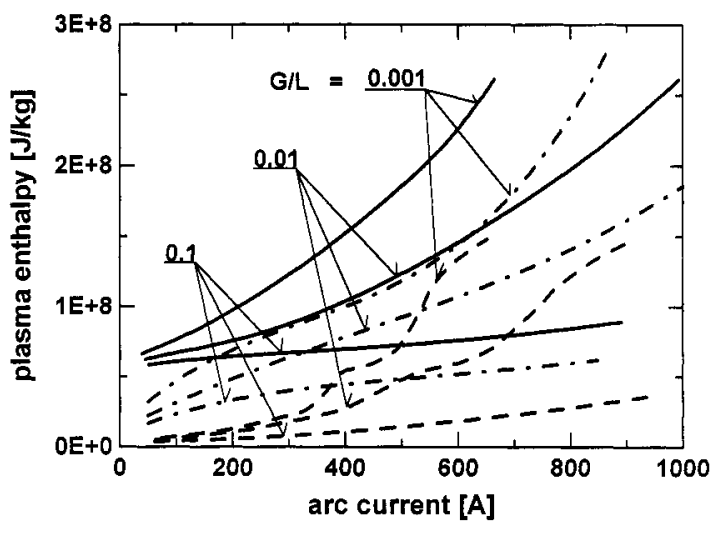

Fig. 3 Dependence of mean plasma enthalpy on the arc current for different ratios of gas flow rate $G$ to the arc length $\mathrm{L}$ for arc in water (full line), nitrogen (dash-and-dot line) and argon (dash line), $\mathrm{R}=3 \mathrm{~mm}$.

Figs. 2 and 3 show curves calculated from (2) and (3) for three plasma mediums - argon. nitrogen and water. LTE values of $\sigma(T), h(T)$ and $S(T)$ were determined using computer ode ADEP (ref. 10), values of net emission coefficient $\varepsilon_{n}$ were taken from (ref. 11,12). It can be seen that stabilization of arc by water leads tr high electric field intensities and thus to high arc powers, and aiso to high plasma inthalpies. However, principle difference of water-stabilized and ges-stabilized arcs ollows from the efiect of ratio $G / L$ on arc characteristics. Due to possibility of higher thermal loading of the wall in water-stabilized plasma torches an arc cain be stabilized with very low values of ratio $\mathrm{G} / \mathrm{L}$. For water torches, values $G / L$ of order $0.001 \mathrm{~kg} / \mathrm{m} . \mathrm{s}$ are typical while for gas-stabilized torches minimum possible values $G / L$ are more than one order higher. Thus, very high plasma enthalpy and temperature can be achieved in water-stabilized torches. This is illustrated in Table 1, where typical parameters for several plasma spraying torches are presented. Values of mean plasma enthalpy and temperature for gas-stabilized torches were estimated assuming $60 \%$ torch efficiency and using thermodynamic properties of plasma gas calculated on the basis of computer code ADEP (ref. 10).

TABLE 1. Typical parameters for plasma spraying torches. Values for mixture $\mathrm{Ar} / \mathrm{H}_{2}$ for two ratios of gas flow rates correspond to plasma torch Plasma-Technik F 4, values for $\mathrm{N}_{2} / \mathrm{H}_{2}$ are for torch PlazJet produced by TAFA (ref. 13).

\begin{tabular}{c|cccccc}
\hline $\begin{array}{c}\text { Plasma } \\
\text { medium }\end{array}$ & $\begin{array}{c}\text { arc current } \\
{[\mathrm{A}]}\end{array}$ & $\begin{array}{c}\text { arc pover } \\
{[\mathrm{kW}]}\end{array}$ & $\begin{array}{c}\text { mass flow rate } \\
{[\mathrm{g} / \mathrm{s}]}\end{array}$ & $\begin{array}{c}G / \mathrm{L} \\
{[\mathrm{kg} / \mathrm{s} . \mathrm{m}]}\end{array}$ & $\begin{array}{c}\text { mean enthalpy } \\
{[\mathrm{MJ} / \mathrm{kg}]}\end{array}$ & $\begin{array}{c}\text { m.temperature } \\
{[K]}\end{array}$ \\
\hline $\begin{array}{c}\mathrm{Ar} / \mathrm{H}_{2} \\
(65 / 3 \mathrm{slpr})\end{array}$ & 750 & 44 & 1.93 & 0.15 & 13.5 & 12100 \\
$\begin{array}{c}\mathrm{Ar} / \mathrm{H}_{2} \\
(33 / 10 \mathrm{slpm})\end{array}$ & 500 & 25 & 0.98 & 0.08 & 15.3 & 10800 \\
$\begin{array}{c}\mathrm{N}_{2} / \mathrm{H}_{2} \\
(235 / 94 \mathrm{slpm})\end{array}$ & 500 & 200 & 5.0 & 0.1 & 24 & 6200 \\
water & 300 & 54 & 0.20 & 0.004 & 157 & 13750 \\
water & 600 & 133 & 0.33 & 0.006 & 272 & 16200 \\
\hline
\end{tabular}




\section{CHARACTERISTICS OF WATER-STABILIZED PLASMA TORCH}

\section{Characteristics of arc}

This paragraph presents results of experiments performed with water stabilized plasma torch designed for power range from 90 to $200 \mathrm{~kW}$ (ref. 14,15). The stabilizing chamber of the torch was created by three sections with tangential water injection. The sections were separated by segments determining diameter of water vortex, the exhaust slots were at the both ends of the stabilizing chamber. The cathode was made of a small piece of zirconium fixed in copper rod, the anode in the form of rotating internally cooled cooper disc was positioned outside of the arc chamber $2 \mathrm{~mm}$ downstream of the nozzle exit. The length of the stabilized part of arc column was $55 \mathrm{~mm}$, the inner diameter of water vortex was $7 \mathrm{~mm}$, the diameter of exit nozzle was $6 \mathrm{~mm}$. Total flow rate of water through the chamber was $301 / \mathrm{min}$.

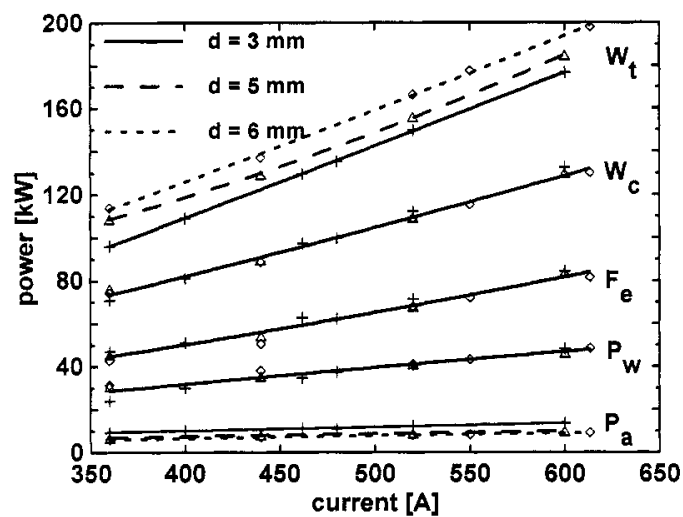

Fig. 4 Power balance of water-stabilized plasma torch for several distances $d$ of surface of anode from the axis of arc.

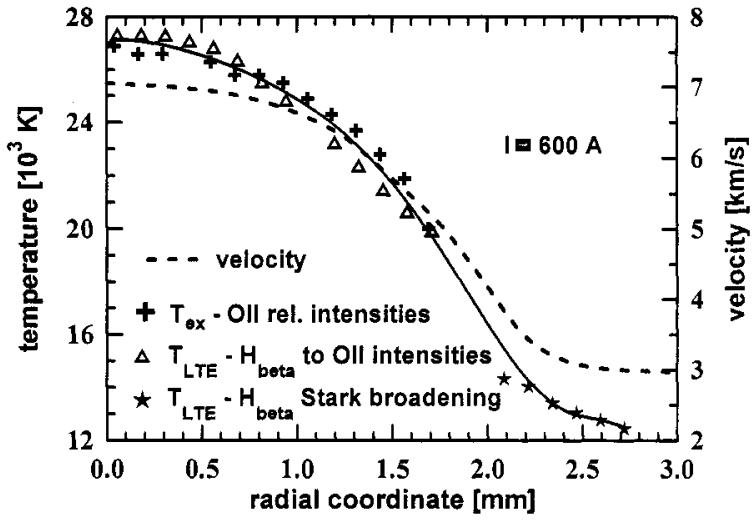

Fig. 5 Radial profiles of plasma temperature and velocity $2 \mathrm{~mm}$ downstream of the nozzle exit for $d=5 \mathrm{~mm}$.

Fig. 4 shows power balance of plasma torch. $\mathrm{W}_{\mathrm{t}}$ is total power input, $\mathrm{W}_{\mathrm{c}}$ power input into water-stabilized part of arc column, $P_{w}$ power loss to the water, $P_{a}$ power loss to the anode and $F_{e}$ total enthalpy flux

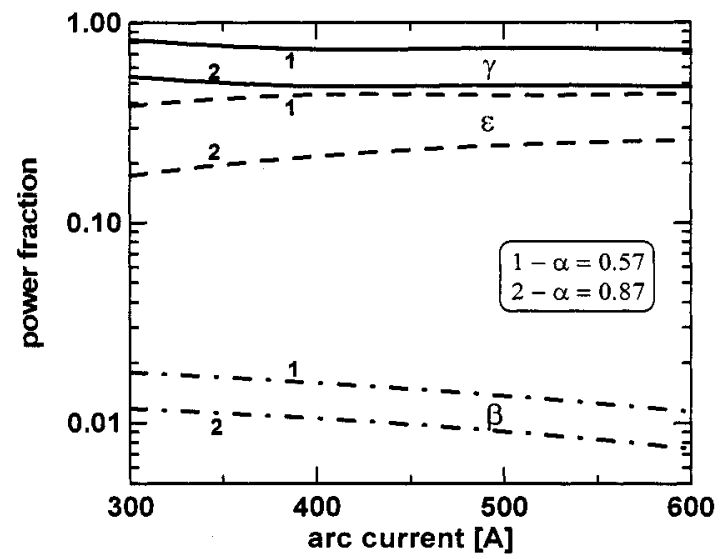

Fig. 6 Balance of radial transfer of energy in the arc column. $\alpha$ is fraction of total arc power transferred in radial direction, $\varepsilon$ fraction of this radiant power absorbed in the vapor sheath, $\beta$ fraction of radiant power spent for evaporation and $\gamma$ is fraction of radiant power absorbed in the water. through the exit nozzle (ref. 14). The profiles of plasma temperature and axial flow velocity $2 \mathrm{~mm}$ downstream of the torch exit are presented in Fig. 5 (ref. 15). Plasma temperature close to $28000 \mathrm{~K}$ and velocity $7 \mathrm{~km} / \mathrm{s}$ were found in the experiments.

Coefficients characterizing balance of radial transfer of energy in the arc chamber are shown in Fig. 6. The coefficients were evaluated from the series of experiments on water stabilized torch (ref. 14). It can be seen that $57 \%-87 \%$ of arc power is transferred in radial direction, about $20 \%(=\alpha . \varepsilon)$ of arc power is absorbed in vapor sheath, only approximately $1 \%$ of arc power is spent for evaporation and power loss to the water is about $40 \%$. Thus, very low amount of energy is absorbed in the boiling layer and spent for evaporation. It is principle cause of low mass flow rate and high plasma temperature and enthalpy in water plasma torches. 


\section{Properties of generated plasma iet}

Basic characteristics of plasma jet measured at the torch exit are shown in Table 2. The jet is characterized by very high plasma temperatures, enthalpies and flow velocities. High flow velocity combined with very low ratio S of plasma density to the density of ambient air leads to intensive turbulent mixing of flowing plasma with ambient gas. Characteristic frequencies of the production of turbulent eddies due to entrainment of cold gas into jet given in the table were determined from power spectra of light emitted from the jet (ref. 16). High characteristic frequencies are related to high flow velocities. The nondimensional frequencies given by Strouhal number $S t=f . D / v_{m}$ are close to the values found in jets produced in gas-stabilized torches with substantially lower flow velocities.

TABLE 2. Torch operating parameters and jet exit conditions.

\begin{tabular}{lcccc} 
arc current $(\mathrm{A})$ & 300 & 400 & 500 & 600 \\
\hline power input $(\mathrm{kW})$ & 84 & 106.8 & 139 & 176 \\
mass flow rate $(\mathrm{g} / \mathrm{s})$ & 0.204 & 0.272 & 0.285 & 0.325 \\
mean temperature $(\mathrm{K})$ & 13750 & 14500 & 15400 & 16200 \\
centerline temperature $(\mathrm{K})$ & 19000 & 23000 & 26200 & 27200 \\
mean enthalpy $(\mathrm{MJ} / \mathrm{kg})$ & 157 & 185 & 230 & 272 \\
mean velocity $(\mathrm{m} / \mathrm{s})$ & 1736 & 2635 & 3247 & 4230 \\
centerline velocity $(\mathrm{m} / \mathrm{s})$ & 2494 & 4407 & 5649 & 7054 \\
mean density $\left(\mathrm{kg} / \mathrm{m}^{3}\right)$ & $4.15 \times 10^{-3}$ & $3.64 \times 10^{-3}$ & $3.1 \times 10^{-3}$ & $2.72 \times 10^{-3}$ \\
centerline density $\left(\mathrm{kg} / \mathrm{m}^{3}\right)$ & $1.92 \times 10^{-3}$ & $1.23 \times 10^{-3}$ & $0.98 \times 10^{-3}$ & $0.92 \times 10^{-3}$ \\
mean density ratio S & 0.0034 & 0.0030 & 0.0026 & 0.0023 \\
characteristic frequency $(\mathrm{kHz})$ & 52 & 68 & 96 & 118 \\
Strouhal number St & 0.18 & 0.15 & 0.18 & 0.17 \\
Reynolds number Re & 473 & 786 & 1140 & 1770 \\
Mach number M & 0.317 & 0.445 & 0.505 & 0.617 \\
\hline
\end{tabular}

The jet is fully turbulent and it is characterized by a presence of large scale structures with very high gradients of density (ref. 17). Intensive turbulent mixing causes rapid decrease of temperature and velocity along the jet. Isotherms of plasma jet generated at arc current $400 \mathrm{~A}$ are shown in Fig. 7 , development of centerline velocity along the jet is shown in Fig. 8. The temperatures were obtained by series of spectroscopic measurements (ref. 15), the velocities were determined by electric probe diagnostics (ref. 18). Despite of their rapid decrease with increasing distance from the torch exit, both the temperature and the velocity are high in the jet.

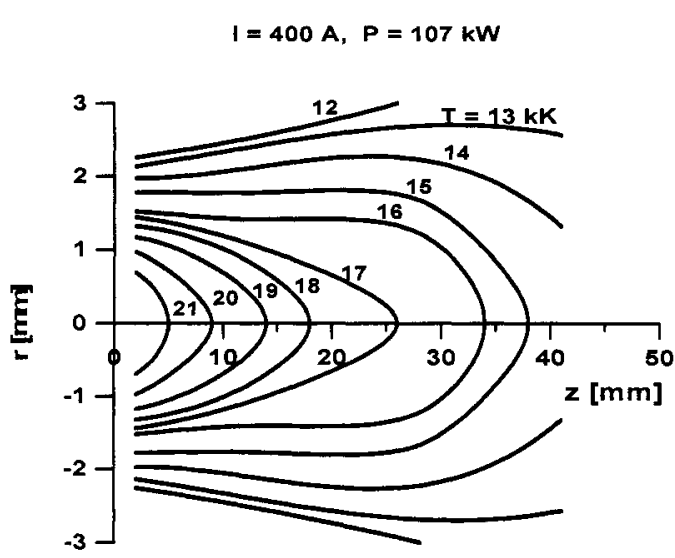

Fig. 7 Isotherms of plasma jet generated in water torch at arc current $400 \mathrm{~A}$ and arc power $107 \mathrm{~kW}$.

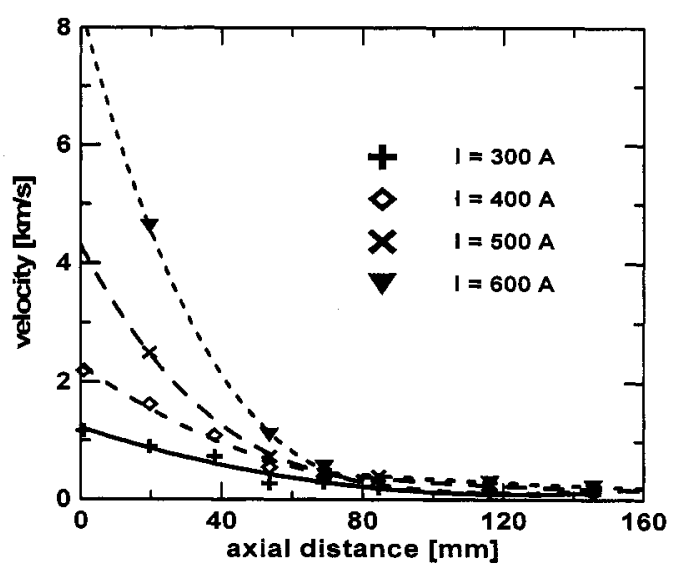

Fig. 8 Development of centerline velocity along the plasma jet. 


\section{CONCLUSIONS}

Principle of stabilization of arcs by liquid wall can be utilized for stabilization of long arc columns at very low flow rates of plasma gas. Thus, plasma jets with high temperatures can be generated in torches with liquid stabilization. In water plasma torches the oxygen-hydrogen plasma is produced, high content of hydrogen results in high enthalpy and sound velocity of plasma. Water-stabilized plasma torches thus generate plasma jet with extremely high plasma temperature and flow velocity.

Basic parameter which determine arc and plasma characteristics is mass flow rate through the arc chamber. This parameter can not be controlled independently as it is determined by power balance of radial transfer of energy in the arc chamber. In water plasma torch the energy spent for evaporation is substantially lower than the energy absorbed in the produced vapour. This is principle cause of low mass flow rates and high temperatures found in experiments. High velocities in combination with low plasma densities lead to intensive interaction of plasma jet with surrounding atmosphere and to high level of turbulence. The mixing of plasma with surrounding cold gas is characterized by very low time constants.

High enthalpy and flow velocity of generated plasma, high level of turbulence and short time constants of mixing result in special performance characteristics in some plasma processing applications. Especially high throughputs of treated material and high process rates are characteristic for water plasma torches. At present the only industrial scale application of water-stabilized plasma torch is plasma spraying. Powder throughputs almost one order higher than for common gas stabilized torches can be achieved (ref. 19). Therefore water-stabilized systems are used for large-area coatings, for production of self-supporting ceramic parts and for powder processing. Application for destruction of liquid waste is investigated (ref. 20,21 ). Besides high level of turbulence, high rate of mixing process and high plasma enthalpy also chemical composition of plasma is advantageous for this application.

\section{ACKNOWLEDGMENTS}

The author would like to thank to the Grant Agency of Czech Republic for the support of this work under the projects No. 102/95/0592 and No. 106/96/K245.

\section{REFERENCES}

1. H. Gerdien, A. Lotz. Wiss. Veroffentlichungen Siemenswerk 2, 489 (1922).

2. H. Gerdien, A. Lotz. Z. Tech. Phys. 4, 157 (1923).

3. H. Maecker. Z. f. Phys. 129, 108-122 (1951).

4. F. Burnhorn, H. Maecker. Z. f. Phys. 129, 369-376 (1951).

5. R. W. Larentz. Z. f. Phys. 129, 343-364 (1951).

6. R. Weiss. Z. f. Phys. 138, 170-182 (1954).

7. F. Burnhorn, H. Maecker, T. Peters. Z. f. Phys. 131, 28-40 (1951).

8. B.Gross, B. Grycz, K. Miklossy. Plasma Technology, Iliffe Books Ltd., London (1968).

9. B.W. Swanson. In Current Interruption of High-Voltage Network (K.Ragaller, ed.), pp. 137-179. Plenum Press, New York London (1978).

10. ADEP - Data bank and computer code. LMCTS URA 320 CNRS, Université de Limoges.

11. H. Riad, J.J. Gonzales, A. Gleizes. Proc. of ISPC 12, pp. 1731-1736. Minneapolis, Aug. 21-25, 1995.

12. V. Aubrecht, B. Gross, J. Phys. D: Appl. Phys. 27, 95-100 (1994).

13. G. Irons. Proc. of Workshop on Industrial Applications of Plasma Chemistry, Vol. B, pp. 53-65. Minneapolis, Aug. 25-26, 1995.

14. M. Hrabovský, M. Konrád, V Kopecký, V. Sember. IEEE Trans. on Plasma Science, in press.

15. M. Hrabovský, V Kopecký, V. Sember. In Heat and Mass Transfer under Plasma Conditions (P. Fauchais, M. Boulos, J. van der Mullen, ed.), pp. 91-98. Begell House, New York-Wallingford (1994).

16. M. Hrabovský, M. Konrád, V Kopecký, J. Hlína. . Proc. of ISPC 12, pp. 1627-1632. Minneapolis, Aug. 21-25, 1995.

17. M. Hrabovský, M. Konrád, V Kopecký, J. Kravárik, P. Kubes. Proc. of $17^{\text {th }}$ Symp. on Plasma Physics and Technology, pp. 179-181. Prague, June 13-16 1995.

18. M. Hrabovský, M. Konrád, V Kopecký. In Heat and Mass Transfer under Plasma Conditions (P. Fauchais, M. Boulos, J. van der Mullen, ed.), pp. 61-67. Begell House, New York-Wallingford (1994).

19. P.Chráska, M. Hrabovský. Proc. of Int. Thermal Spray Conf. \& Exhib., pp. 81-85. Orlando, USA, May 28-June 5, 1992.

20. V. Brožek, M. Hrabovský, V. Kopecký. Proc of ISPC 13, pp.1735-1739. Beijing, August 18-22, 1997.

21. V. Brožek, M. Hrabovský, V. Kopecký. Journ. of High Temp. Mat. Process, in press. 